
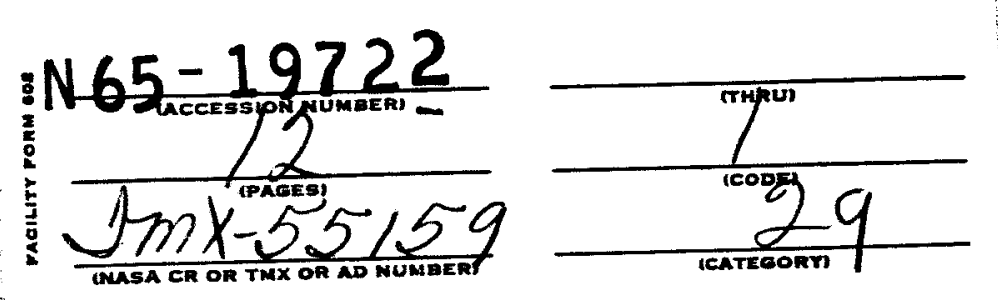

$x-611-64-362$

IASA +AY-55/54

has

\title{
DETECTION OF INTERPLANETARY 3- TO 12-MEV ELECTRONS
}

\author{
T. L. CLINE \\ G. H. LUDWIG \\ F. B. MCDONALD
}

NOVEMBER 1964

NASA

GODDARD SPACE FLIGHT CENTER

GREENBELT, MARYLAND 
DETECTION OF INTER PLANETARY 3- TO 12-MEV ELEĆTŔNONS

T. L. Cline, G. H. Ludwig, and F. B. McDonald NASA-Goddard Space Flight Center, Greenbelt, Maryland

In this Letter we report the direct observation of interplanetary electrons of energy above $3 \mathrm{KeV}$ with the MP-1 satellite (Bxplorer 18).

Blectrons observed in the primary interplanetary radiation in the BeV energy region by $\operatorname{Earl}^{1}$ and in the $200-\mathrm{MeV}$ energy region by Meyer and Vogt ${ }^{2}$ are believed to be of galactic origin because their energies are as high as those assumed to be necessary for their penetration into the inner solar system and because their measured intensity agrees with that which was anticipated to account for galactic radio enission. Support was lent to this hypothesis when the modulation characteristics of these particles were observed ${ }^{3}$ to be similar to those of cosmic-ray protons and their positron-to-electron ratio was found 4 to be compatible with an origin of at least half of them in meson-producing cosmic-ray interactions in the interstellar medium. He feel that the existence of an interplanetary flux of electrons lower in energy by orders of magnitude is interesting because of the possibility that these too may have a cosmic origin. If so, their study should yield entirely new information about the galactic electron sources and modulation characteristics. If they are of solar origin, there are analogous implications. We wish to demonstrate here that the flux of lower-energy electrons we observe is indeed a primary component of the interplanetary radiation, and to discuss its properties in terms of its possible origin, either galactic or solar.

The observations reported here were made with a scintillator telescope on Explorer 18, a satel1ite placed in an elliptic orbit with an apogee height of 
193,000 kilometers. Data were taken from the launch, on 27 November 1963, until 6 May 1964 when the satellite passed into a long period in the earth's shadow, causing failure of the detector. During this time interval the apogee moved from the sunlit side of the earth beyond the magnetosphere (terminating at about $70,000 \mathrm{~km}$ ) and beyond the earth's shock front (observed with a magnetometer ${ }^{5}$ and plasma sensor 6 at about $100,000 \mathrm{~km}$ ) to the region behind the earth and inside the shock front. Electron data taken only when the satellite was beyond 125,000 kilometers are reported here; throughout the life of the instrument these data continued to be free from effects due to the trapped radiation.

The detector was developed to study low-energy cosmic-ray protons, electrons and light nuclei. It is composed of three scintillators: two in coincidence, measuring energy loss and total energy, and a guard counter in anticoincidence. When a table of intensity vs. measured energy loss vs. measured total energy is constructed from data taken at apogee, there is seen a distinct counting rate component of minimum-ionizing energy loss and of low apparent energy. An analysis of the topology of distributions in energy loss and in total energy through this minimum-ionizing component indicates that indeed it is composed of three distinct particle groups: One group with total energies correspording to electrons that stop within the detector, a much smaller group with a high apparent total energy equal to or exceeding the energy loss of a minimumionizing cosmic ray traversing the detector, and a third group with very low total energies. We believe that the latter two components are surely secondary radiations composed of, respectively, cosmic rays that avoid detection by the 
guard counter (for example, by turning into neutrals through reactions within the detector) and gamma rays made locally in the spacecraft, producing random and coherent coincidences between the energy loss and total energy detectors. These secondary effects were eliminated to produce Figure 1a, which shows the energy spectrum of electrons obtained during the first orbit (27 to 30 November 1963) at a time when the observed electron intensity was at a typical minimum and when there were no measurable time variations. Figure 1b shows, for comparison, a spectrum of the difference between the first statistically significant intensity increase (13 to 16 Jamuary 1964) and the immediately preceding intensity ( 9 to 12 January). No background corrections were necessary to produce the latter distribution since the electron intensity increase was unaccompanied by an increase of either secondary gamma rays or spurious cosmic rays; it was therefore possible to determine the intensity to higher energies. The nearly identical shapes of the two corrected spectra suggest that the electrons seen daily may have the same origin as the extra ones seen on days of increased electron flux. The integral intensity of electrons of energy between 2.7 and $7.5 \mathrm{MeV}$ is $210 . \pm 10$. electrons/m $/ \mathrm{m}^{2}$ sec.ster., and that of the increase between 3. and $12.5 \mathrm{MeV}$ an additional $100 . \pm 10$. electrons/m ${ }^{2}$ sec.ster. To demonstrate that most of the observed electrons are not of local or secondary origin at the satellite (e.g., such as knock-on or cascade-shower electrons produced in or near the detector) we consider their time variations. Figure 2 shows the counting rate of these electrons, partially corrected for slow gain drifts in the detector, plotted in the form of one-quarter-orbit 
averages throughout the active life of the instrument. (The gaps in the data occur at times when the satellite is within 125,000 kilometers; the other three points per orbit are plotted so that each center one represents data taken from beyond 185,000 kilometers.) Also shown are a comparison plot "C" of the integrated cosmic-ray flux into a scintillator with about $0.3 \mathrm{gram} \mathrm{cm}^{-2}$ shielding, and the times of a recurrent minimum in the interplanetary magnetic activity index $k_{p}$ with a period of one solar rotation.

A dominant feature of the electron rate is the appearance of many statistically meaningful intensity increases, including one series apparently coincident with the recurrent $\mathrm{K}_{\mathrm{p}}$ minimum. These electron intensity increases were not accompanied by comparable increases in the integral cosmic-ray intensity above $15 \mathrm{MeV}$ : the magnitude of the electron modulation is 50 per cent on occasions, while the cosmic rays undergo modulations of less than 5 per cent. Further, following the flare of 16 March 1964 there was a solar-proton event, accompanied by Type IV solar radio emission ${ }^{8}$, during which the flux of protons of energy between 15 and $75 \mathrm{MeV}$ briefly increased by several orders of magnitude, while the 3- to 8-MeV electron flux rose less than $50 \pm 25$ per cent. (Figure 2 shows the quarter-orbit average of the total integrated cosmic ray flux increasing at that time by about 10 per cent.) These comparisons demonstrate that, at most, an insignificant fraction of the electron modulation results from modulations of cosmic rays of energy above $15 \mathrm{MeV}$.

Modulations of protons with energies below $15 \mathrm{MeV}$, such as 27-day recurrent solar proton events similar to those observed ${ }^{9}$ with Explorer 12 , were not monitored with our apparatus; but these would be expected at the times of 
recurrent Forbush decreases and geomagnetic activity, rather than at the time of our repeating electron increases. Several such 1- to 10-MeV proton intensity increases were observed early in the life of the satellite by Fan, Gloeckler and Simpson 10 but these were about two weeks out of phase with our electron enhancements and appear to be accompanied by, if anything, decreases in the electron intensity and in the galactic cosmic rays.

Finally, a study of 3-hour averages of the observed intensity of these electrons indicates no variation with distance from the earth, either during orbits of minimum intensity or during times of increased intensity; the electron rate is constant through the shock front to a distance of up to 50 per cent beyond 1t. Further, the satellite's passage through the wake of the moon 5 was unaccompanied by an electron intensity varlation. Thus, these electrons are not secondary to cosmic rays or solar protons or due to geophysical processes.

We feel that the question of whether these primary electrons originate at the sun or in the galaxy cannot be definitely answered on the basis of the available data; however, the following properties of these electrons are consistent with their being galactic. First, the differential energy spectrum of this 3- to 12-MeV component fits smoothly onto a spectral plot of the cosmicray electron intensities ${ }^{1,2,4}$ at much higher energies. Second, the time variations of the electrons can be compared to those of cosmic rays in that there is a strong correlation between the electron intensity increases and quiet interplanetary conditions, evidenced by $k_{p}$ minima and very small sea-level cosmic-ray intensity increases. Third, there appears to be a long-term increase 
$\sigma \tilde{f}$ electron intensity after a correction of the same order is applied for a slow, monitored drift in detector gain; if this increase is real; it is similar to the 11-year modulation of cosmic rays as solar minimum is approached. However, the fact that the differential cosmic-ray proton intensity is peaked at about $1 \mathrm{BV} / \mathrm{C}$ rigidity and negligible below $150 \mathrm{MV} / \mathrm{c}$ markedly contrasts with the fact that electrons of rigidity $\gtrsim 3.5 \mathrm{NV} / \mathrm{c}$ are more abundant than those of greater rigidity. Parker has recently pointed out ${ }^{11}$ that particles with gyroradius close to the idealized irregularity scale of the modulating mediun should be deflected wore than those of either extreme; thus these electrons of low rigiuity might wiginate in the galaxy and penetrate the solar system as easily as those of great rigidity.

In spite of the foregoing arguments for galactic origin, it is not impossible that the electrons cane instead from the sun. Several possibilities present thenselves. For example, relativistic electrons might be generated over most of the upper surface of the solar atmosphere, in thich case regions of enhanced and expanded plasma (which contain recurrent proton fluses) would tend to contain fewer electrons while regions of quiet-time streaming would contain more, as we have observed. Further, the deceleration of the electrons in the enhanced plasma night be much greater than that in the quiet-time strcaming. Aiternatively, the electrons right be associated with the development of new sunspot regions, which is a characteristic of this phase of the solar cycle and appears to corcelate weakly with the observed pattern of intensity increases.12 we have not, however, found a corralation with any solar radio or opticil activity. 


$$
-7-
$$

The results we quote here are preliminary: an evaluation of the detector response, providing a more exact spectrum, and a detailed investigation of the time variations w111 be given elsewhere. We are happy to acknowledge the efforts of the many people who made the MiP-1 a success. 


\section{REFERENCES}

1. J. A. Barl, Phys. Rev. Letters 6 , 125 (1961).

2. P. Meyer and R. Vogt, Phys, Rev. Letters $\underline{\epsilon}, 193$ (1961).

3. P. Meyer and R. Vogt, J. Geophys. Res. 66, 3950 (1061).

4. J. A. DeShong, Jr., R. H. Hildebrand and P. Meyer, Phys. Rev. Letters 12, 3 (1964).

5. N. F. Ness, C. S. Scearce and J. B. Seek, Space Research 5, N. Holland Pub1. (COSPAR Proceedings, Florence, 1964).

6. H. S. Bridge, A. Bgidi, A. Lazarus, B. F. Lyon and I. Jacobson, Space Research 5, N. Holland Pub1. (COSPAR Proceedings, Florence, 1964).

7. D. A. Bryant, G. H. Ludwig and F. B. McDonald, I.R.E. Transactions on Nuclear Science NS-9, 376 (1962).

8. A. Maxwe11, private communication.

9. D. A. Bryant, T. L. Cline, U. D. Desai and F. B. McDonald, Phys. Rev. Letters 11, 144 (1963).

10. C. Y. Fan, G. Gloeckler and J. A. Simpson, Goddard IMP Symposium, March 1964.

11. E. N. Parker, J. Geophys, Res. 69, 1755 (1964).

12. H. Dodson Prince and R. Hedeman, private communication. 


\section{F IGURE CAPTIONS}

FIG. 1. Differential energy spectra of electrons observed beyond 125,000 kilometers from the earth. The first spectrum is from the apogee of the first orbit; the second is the difference between measurements from the 13 th and 12 th orbits and indicates the first significant increase in intensity.

FIG. 2. Integral counting rate of electrons throughout the active life of the instrument plotted in quarter-orbit averages. The counting rate " $\mathrm{C} "$ of cosmic rays into a thinly shielded scintillator and the times of one recurrent minimum of the interplanetary index $\mathbf{F}_{\mathbf{p}}$ are also shown. Recurrent Forbush decreases are seen in the cosmic rays in early December and January and a small solar proton event occurs in March; other increases can be largely attributed to the electron mixture in the cosmic rays. 


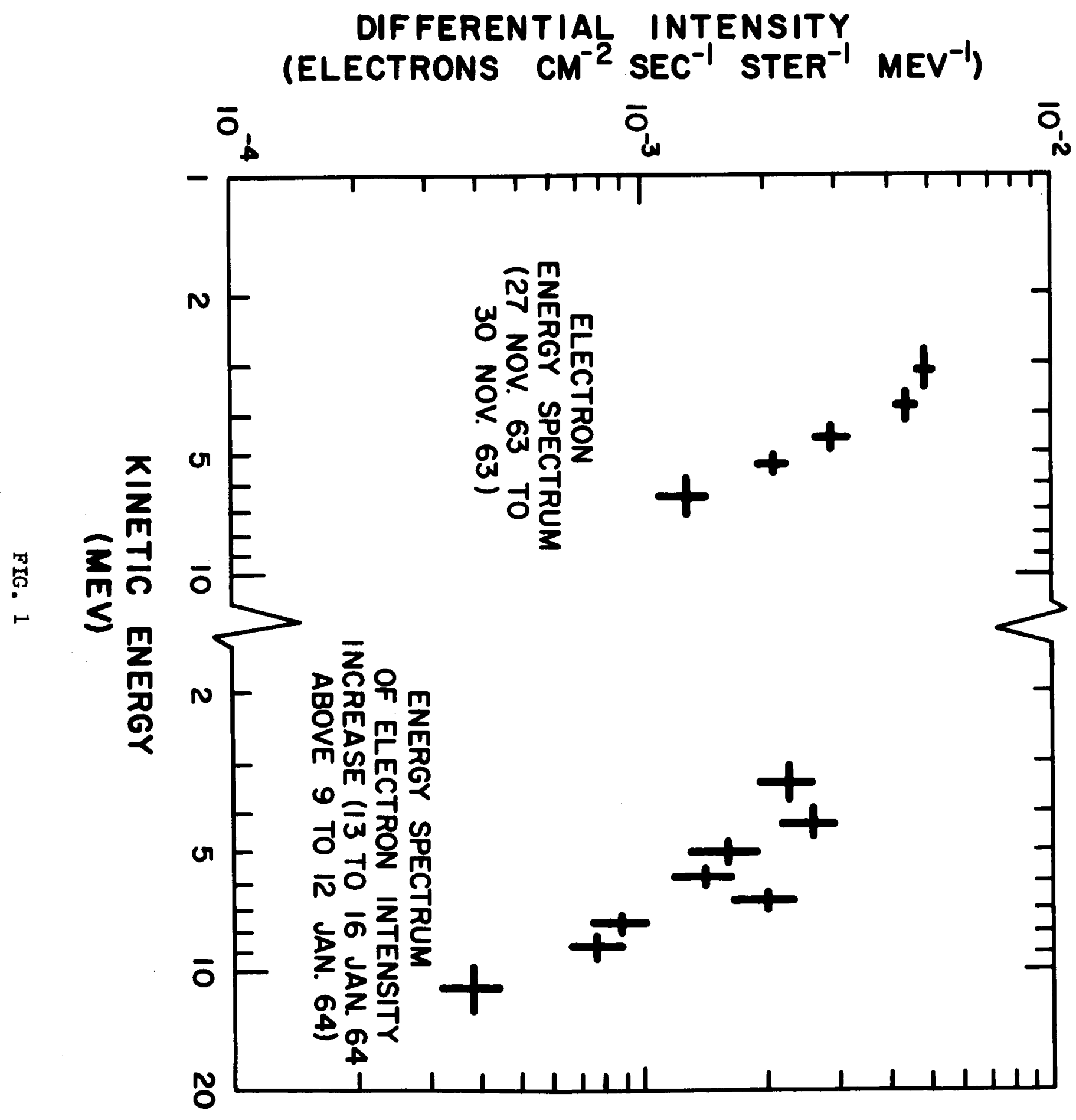




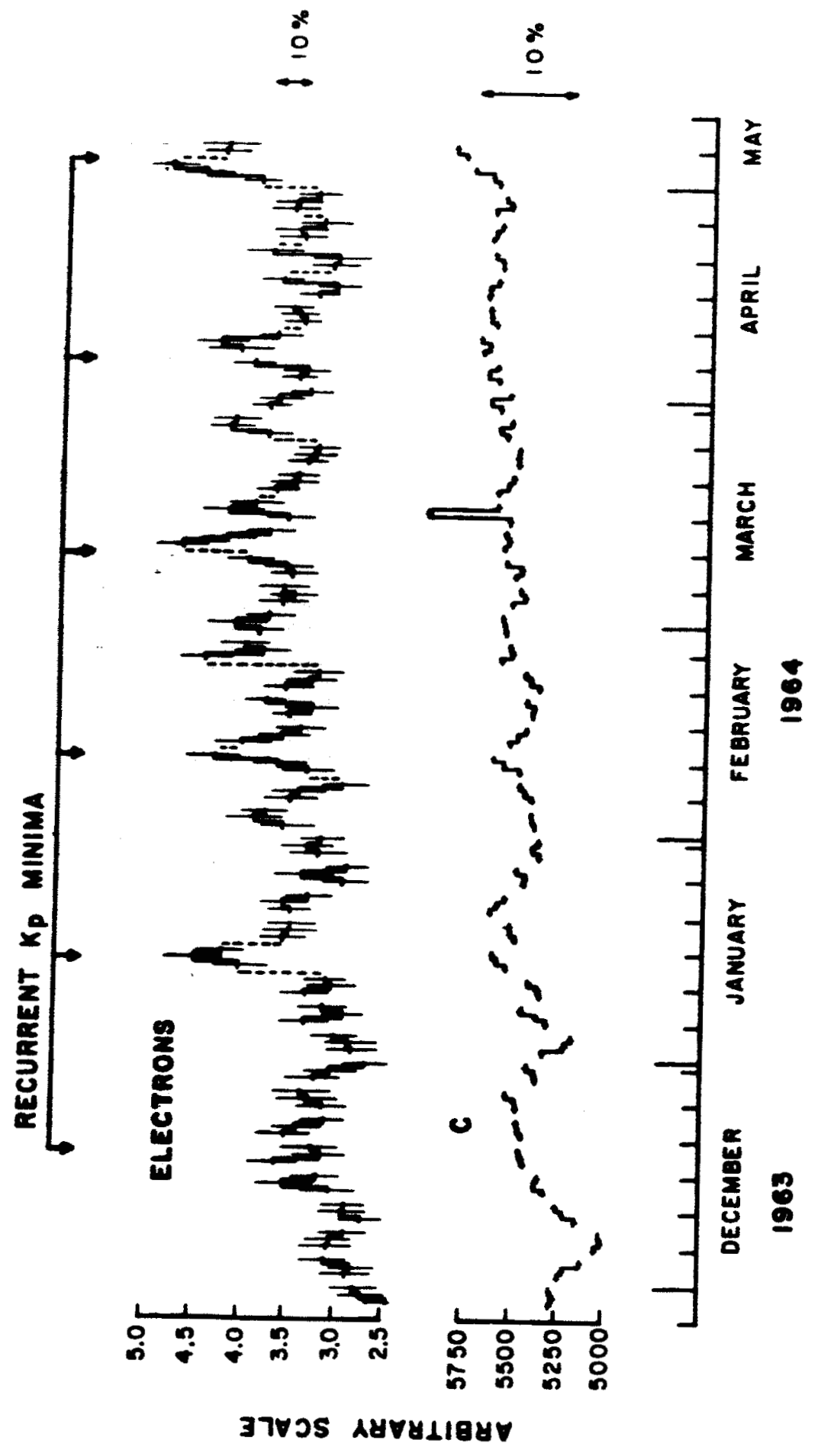

